\title{
TRÊS MARCOS NA CONSTRUÇÃO DO PROJETO ADOLESCENTE: HISTÓRIA PESSOAL; CULTURA; ASSOCIAÇÃO*
}

\author{
THREE LANDMARKS IN THE CONSTRUCTION OF THE \\ ADOLESCENT PROJECT: PERSONAL HISTORY; \\ CULTURE; ASSOCIATIONS
}

Emile-Henri Riard ${ }^{1 *}$

RESUMO: Tendo como referencial teórico a concepção de BLOS e MAHLER sobre o desenvolvimento (processo de separação/individuação), bem como os aportes de Kaës quanto aos conceitos de filiação/afiliação (KAËS, 1985), de transicionalidade (KAËS, 1979) e de ideologia (KAËS, 2016), o presente artigo chama a atenção para três elementos envolvidos na construção do projeto de vida de adolescentes franceses, cujas famílias são oriundas de meios culturalmente distintos. Tais elementos aparecem, de modo recorrente, em pesquisas que realizamos sobre o tema, desde 1997. São eles: a dimensão imaginária ligada ao processo adolescente, muitas vezes negligenciada em abordagens institucionais; as repercussões das dificuldades dessa construção para os jovens confrontados por várias culturas e seu entorno; e, finalmente, o lugar que as associações de vizinhança podem ter nesse processo e suas implicações conceituais.

Palavras-chave: Adolescente. Cultura. Associações. Corpos psíquicos. Transicionalidade e filiação.

ABSTRACT: The imaginary dimension, multicultural situations and the role of neighbourhood associations are often overlooked in research on the construction of the adolescent project. This article aims to identify the impact on young people living in France, from culturally differentiated backgrounds. The theoretical framework is that of BLOS and MAHLER (separation/individuation), supplemented by KÄ̈S' contributions on the concepts of transitionality (1979), filiation/affiliation (1985); ideology (2016), which have been the result of searches conducted since 1997.

Keywords: Adolescent project. Culture. Associations. Psychic agency. Transitionality and filiation.

1.Université Picardie Jules Verne - Laboratório CAREF - Amiens, França.

*Autor correspondente: eh.riard@laposte.net

Dossiê organizado por: Joyce Mary Adam e Débora Cristina Fonseca

-Este artigo é tradução do original em francês "Trois Repères Dans La Construction Du Projet Adolescent: Histoire Personnelle; Culture; Associations", (também disponibilizado online) feita por Mary Joyce Adam e revisada por Izabel Galvão 


\section{Introdução}

A adolescência é, para os jovens que a atravessam, tempo de profundas e decisivas alterações psíquicas. Nessa etapa da vida, os adolescentes têm que abandonar as identificações da infância e olhar para o futuro, a fim de acessar um tempo pessoal, o tempo da construção da identidade. É nesse momento que surge, para eles, a questão do projeto de vida "naturalmente" orientado para o futuro: seu conteúdo e os meios necessários para alcançá-lo. O projeto é um vetor para os adolescentes atingirem o status de adulto e, consequentemente, sua futura integração social e profissional. Ele concerne, num processo associado a um conjunto de valores, aos dois elementos inseparáveis de todo o funcionamento humano: o da realidade e o do imaginário, sendo o último ausente ou, pelo menos, negligenciado nas abordagens que a sociedade - especialmente a escola - tem desse processo. Além disso, essa construção, dependente de mudanças adolescentes de todos os tipos (somática, psíquica, social), é sempre realizada em um momento particular da história da família e do grupo de jovens, em uma área cultural dada. Esse contexto tem grande impacto nessa construção.

O objetivo deste artigo é apresentar três elementos que, embora sejam frequentemente negligenciados pelas pesquisas, desempenham papel fundamental na construção do projeto adolescente, a fim de analisar o impacto sobre ele: 1) a história pessoal do sujeito, com foco na dimensão imaginária e em sua relação com a realidade; 2) a cultura, levando em conta, sobretudo, o lugar que ocupa a herança recebida, essencialmente dos pais dos jovens (herança de comportamentos, valores, códigos), o que suscita a questão da filiação e da afiliação, bem como das dificuldades quando o código cultural (parental) recebido não corresponde ou corresponde apenas parcialmente ao da sociedade de residência do jovem; e 3) por fim, as associações de bairro que, frequentadas de modo voluntário pelos jovens, oferecem a eles, em complemento à família e à escola, oportunidades para conseguirem construir seu projeto quando encontram dificuldades que não puderam ser tratadas positivamente por nenhuma dessas duas instâncias.

O conteúdo deste artigo é resultado de pesquisas desenvolvidas no âmbito de diferentes projetos, em parceria com instituições governamentais e não governamentais ${ }^{1}$. As equipes foram compostas por psicólogos (principalmente) e sociólogos. Essas pesquisas também provêm de doutorados em psicologia social clínica, conduzidos sob a direção do autor deste artigo (MAINE, 2009; HOANG, 2012). Elas foram realizadas em ambientes social e culturalmente diversos, principalmente na França e no Marrocos, com base em amostras representativas. O número total de sujeitos considerados neste artigo é 650 . Os sujeitos foram divididos de modo aproximadamente equivalente entre meninas e meninos; por idade (12-13 anos e 11 meses; $14-15$ anos e 11 meses; 16 a 18-20 anos); e por local de residência (rural ou urbana), quando necessário. ${ }^{2}$

Além de um questionário específico a cada pesquisa, incidindo sobre a dimensão da realidade do projeto dos jovens, foi proposto, sistematicamente, um teste de orientação projetiva, cuja função é atualizar os mecanismos do imaginário (mecanismos de defesa específicos, representações, modo de resolução de conflito etc.). Consistia em pedir aos sujeitos que imaginassem uma história baseada em mapas projetivos do Teste de Percepção Temática de Murray (1953). ${ }^{3}$

Após uma breve síntese dos procedimentos utilizados pelas escolas - baseados na aplicação de diretrizes do Ministério da Educação Nacional - para auxiliar os jovens na sua orientação escolar e profissional, será abordado o lugar que a história pessoal do sujeito ocupa na construção do projeto profissional, por meio dos remanejamentos próprios a esse período da vida (1). Em seguida, serão destacadas as repercussões particulares que a história pessoal pode ter para os jovens de outra cultura, particularmente em sua escolha de vida, colocando a questão das relações entre filiação e afiliação (2). Por fim, a última parte proporá uma possível resposta para os jovens que enfrentam dificuldades em construir seu projeto: a fornecida pelos equipamentos do setor associativo (3). ${ }^{4}$ 


\section{A Escola e a Construção do Projeto}

Uma das tarefas essenciais que a escola e as famílias pedem aos jovens na França é construir um projeto, mais frequentemente considerado exclusivamente em sua dimensão profissional. Para esse fim, a escola configura um dispositivo que vai desde o inicio do Ensino Fundamental II até o final do Ensino Médio - de 11-12 anos a 14-15ans. Esse trabalho começa propondo aos alunos uma reflexão sobre seu futuro. Em seguida, apoia-se em testes e entrevistas realizadas por um psicólogo escolar [conseiller d'orientation]. Ao fim do Ensino Fundamental, os jovens podem integrar, quer um Ensino Médio técnico ou profissional, quer uma escola geral de Ensino Médio. Dependendo dos resultados acadêmicos de cada joevm, a orientação para um ramo profissionalizante pode ser proposta antes mesmo do Ensino Médio.

A construção do projeto profissional é, assim, concebida como algo realizado por escolhas preliminares e sucessivas e apoiado em fatores individuais, incluindo os desejos dos jovens. O processo seguido pela escola se inspira em trabalhos científicos que remetem a três concepções bem conhecidas, desenvolvidas sucessivamente por GINZBERG et al. (1951), CRITES (1962) e SUPPER (1988). O primeiro centra-se no desenvolvimento chamado "vocacional": a escolha profissional do adolescente resulta em um processo irreversível, que consiste, em primeiro lugar, de escolhas ditas "fantasiosas", as quais vão se tornando cada vez mais "realistas". CRITES (1962), seguido por SUPPER (1988), introduz a ideia de "maturidade profissional”, baseada em uma mobilização do indivíduo na busca de uma solução que leve em conta suas aptidões, suas motivações etc. e uma cristalização gradual em seus interesses e valores de trabalho para estabelecer uma coerência entre preferências profissionais e autoestima. HUTEAU (1993) ampliou essa visão, colocando-a no terreno das representações, essencialmente familiares e identitárias e vinculando a profissão e a identidade de modo a estabelecer uma relação entre representação de si e representações profissionais. GUICHARD (1993) considera a importância determinante das representações que os adolescentes têm acerca da atividade profissional dos pais sobre seus próprios projetos.

No entanto, outra abordagem, que não invalida as anteriores, tampouco as que serão desenvolvidas ulteriormente, foi esboçada por MÂLE (1962), psiquiatra para quem as escolhas profissionais têm origens arcaicas e edipianas, e, portanto, estariam enraizadas no imaginário dos jovens.

A partir dos referenciais mencionados acima, partindo de uma perspectiva integrativa, propõe-se considerar o projeto profissional não como elemento isolado do resto da vida e dependente unicamente dos elementos específicos, classicamente já mencionados - interesses, atitudes, competências etc. -, mas como parte fundamental de um todo constituído pela pessoa do jovem. Trata-se, portanto, de um único projeto, constituído por diversas facetas, sendo as principais a vida profissional e a vida privada. Essa perspectiva reúne e coloca em interdependência as dimensões emocional, cognitiva, intelectual e social do indivíduo em íntima conexão com seu desenvolvimento. A definição proposta é a seguinte: "o projeto é um ato de engajamento do sujeito no futuro, um fio condutor que entrelaça, numa relação de interdependência, as dimensões manifestas e latentes, emocionais e cognitivas, o passado, o presente e o futuro" (RIARD, 2004, p. 18). Os trabalhos de CASTELLAN e RIARD (2005) e de CASTELLAN (2007) reforçam essa abordagem, a qual constitui a base deste artigo.

\section{História Pessoal do Sujeito}

\section{Referencial e contexto teórico}

As abordagens do desenvolvimento psíquico propostas pelos psicanalistas MAHLER (1982) e BLOS (1967), mantidas aqui e adotadas na maioria dos trabalhos científicos, são denominadas "processo 
de separação/individuação". Segundo elas, o desenvolvimento se dá por meio de duas principais etapas. A primeira corresponde principalmente à primeira infância e consiste principalmente em um trabalho de separação (especialmente físico) da mãe, contendo os germes da individualização. Esse trabalho continua durante o período de latência (dominado pelas dimensões cognitiva, intelectual e social), de modo silencioso. A segunda etapa, que se prolonga por vários anos, é "organizada" pela individualização, pela construção da identidade. É desencadeada por um crescimento poderoso e brutal dos afetos que acompanham o ressurgimento do complexo de Édipo e traz de volta à superfície muitos materiais psíquicos, tais como representações, mecanismos de defesa, fantasias etc., às vezes muito antigos na história do sujeito, mas frequentemente revistos e corrigidos por sua história mais recente. Essa etapa permite que o sujeito se distancie do mundo da infância, principalmente das figuras parentais e seus representantes. O jovem abandona - ou ao menos deve fazê-lo - a dualidade dependência infantil versus onipotência parental (presente no período de latência). É, portanto, no contexto de múltiplas e profundas reorganizações das relações objetais que se dá o trabalho de construção do projeto, o qual deve permitir que o jovem, em longo prazo, possa acessar e assumir as duas principais funções do estado adulto: a de "produtor", que diz respeito ao mundo social e, em particular, profissional, e a de "reprodutor", relacionada à vida afetiva. Essas duas funções também terão de ser correspondidas por um conjunto de valores.

Vamos agora olhar para o reprocessamento dos materiais psíquicos localizados no âmago do processo de adolescência. Quais são seus conteúdos e as relações entre eles e com a dimensão manifesta do projeto?

\section{Materiais e mecanismos envolvidos na construção do projeto}

A literatura de orientação psicanalítica dedicada ao processo da adolescência é particularmente frutífera e convergente quanto aos materiais presentes e envolvidos nesse processo (KESTEMBERG, 1962; BLOS, 1967; ERIKSON, 1968; GUTTON, 1983; CHABERT, 1993; JEAMMET, 1983; MARCELLI; BRACONNIER, 2007; LESOURD, 2005; GOLSE, 2008). As transformações adolescentes são apresentadas em torno essencialmente de três instâncias psíquicas, que têm a particularidade de serem todas presentes, de um extremo ao outro da vida, garantindo assim a continuidade do sujeito, para além de suas transformações. Essas instâncias são: as identificações (que tangem principalmente o passado infantil e têm por alvo principal os pais); os ideais do eu (que são a expressão de desejos pessoais orientados para o futuro e cuja aparência é correlativa do declínio do superego, esse último de origem parental); e, por fim, o equilíbrio narcísico-objetal, que sofre, nessa época da vida, uma redistribuição da relação entre as dimensões objetais e narcísicas (i. e., entre os lugares respectivos atribuídos ao outro e a si - em particular, ao outro sexo - no funcionamento psíquico dos jovens). A presença desse contrapeso é fundamental na busca de um equilíbrio baseado, doravante, na complementaridade, não mais na mera diferença dos sexos, complementaridade também necessária entre as dimensões cognitiva e afetiva do que é indispensável para o equilíbrio de cada indivíduo. Como e de que maneira esses materiais participam da construção do projeto adolescente?

Para responder a essa pergunta, é necessário adotar como ponto de referência as duas principais formas de projeto identificadas a partir dos resultados das pesquisas realizadas com jovens de 11-12 a 15-16 anos. Trata-se do projeto dito "bem-encaminhado" e do projeto "fraco", às vezes "mágico" (RIARD, 1996; RIARD; DACHMI, 2004).

Em um projeto "bem-encaminhado", o jovem procura ativamente informações sobre a profissão que projeta, busca ajuda, informa-se, abandona racionalmente seus projetos anteriores, dá uma olhada em si mesmo e em suas capacidades e aptidões, leva em conta também seus interesses, bem como concede um lugar 
para os outros (relações de amizade ou amorosas), além de se planejar para um período de aproximadamente dez anos, adotando também um conjunto de valores.

Inversamente, o projeto "fraco" traduz-se, essencialmente, por elementos dispersos, é reduzido frequentemente à dimensão afetiva, e seu autor negligencia toda a busca de informação sobre as profissões, pouco se esforçando para projetar-se no futuro, tampouco se questionando sobre seus interesses e habilidades.

\section{Características do envolvimento do jovem no projeto}

\section{A presença de correspondência entre as dimensões aparente e imaginária do projeto}

Os resultados das pesquisas que cruzam entrevistas e provas de natureza projetiva, do tipo Thematic Apperception Test (T.A.T) (MAINE, 2009; CASTELLAN, 2007; RIARD, 1996; RIARD; DACHMI, 2004), ressaltam a presença de correspondências entre a qualidade do projeto que se observa no nível manifesto e a ressaltada no nível latente. Por exemplo, a um projeto considerado "bem-encaminhado" (ver "Materiais e mecanismos envolvidos na construção do projeto"), corresponde, no nível latente, um trabalho de reorganização de relações objetais orientadas para o acesso ao estado adulto (reprocessamento das identificações da infância; presença de um trabalho de separação e conflitualização das figuras parentais; presença de ideais mais pessoais; e presença de um lugar importante dado ao outro sexo em relações de complementaridade, não simplesmente de diferença). Inversamente, a um projeto manifesto considerado "fraco" (ver "Materiais e mecanismos envolvidos na construção do projeto") corresponde, no nível latente, uma fragilidade na orientação do trabalho psíquico que conduz ao estado adulto, o que ainda mantém seus autores em uma posição de coloração infantil. Isso se expressa por um trabalho de separação e conflitualização fracamente engajado ou inexistente; pela quase ausência de desejos pessoais e por um lugar considerável, até excessivo, atribuído às relações de natureza narcísica; o outro - especialmente o outro sexo - não "existe", salvo para, de algum modo, "segurar o espelho" (i. e., para retornar ao sujeito sua própria imagem).

\section{Natureza sincrônica da qualidade dos componentes das instâncias psíquicas}

Como ressaltado, resulta da característica anterior que o trabalho feito no nível de cada uma das três instâncias vá sempre na mesma direção, ao mesmo tempo. É o que ocorre nos componentes observados no trabalho subjacente ao projeto "bem-encaminhado", todos apresentando qualidades comparáveis ao mesmo tempo: a presença da separação do conflito com as figuras dos pais E a criação de ideais pessoais E diferentes representações do outro sexo numa perspectiva de complementaridade. As três instâncias do imaginário também são de qualidade comparável, no mesmo momento, quando o projeto do jovem é "fraco": impossibilidade da (necessária) conflitualização ou separação das figuras parentais; ausência de ideais pessoais; grande dificuldade ou mesmo incapacidade de se ver em relações de complementaridade com o outro sexo, como se existência desse se justificasse somente para "segurar o espelho".

Essa sincronicidade dos materiais do imaginário remete à questão do modo de funcionamento psíquico.

\section{Construção do projeto em um modo de natureza oscilatória}

A análise dos resultados dessa construção conduz, inicialmente, à hipótese de que os componentes das três instâncias envolvidas podem ser agrupados em polos de relações objetais, com base em seus pontos

Cad. Cedes, Campinas, v. 40, n. 110, p.74-85, Jan.-Mar., 2020 
comuns em termos de distância relacional. Em um dos polos, a qualidade dos materiais coloca o adolescente em um jogo de relacionamentos, com base na busca de diferença/complementaridade (específicas do estado adulto). As relações são caracterizadas por um trabalho de reprocessamento de identificações infantis, que encorajam ideais pessoais e redistribuem as relações entre as dimensões narcísicas e objetais no sentido de buscar a complementaridade com o outro sexo. O outro polo é constituído por materiais que especificam as relações da ordem de proximidade/similitude, que dizem respeito a modos de relações inerentes ao estado infantil - ou próximo dele -, na medida em que o trabalho realizado em direção ao status de adulto é fraco. Essas relações ajudam a manter o sujeito em estado de dependência infantil versus onipotência parental; o outro sexo ainda sendo visto a partir da perspectiva de mera diferença, bem como os desejos pessoais podendo ser inexistentes ou "mágicos".

Na maioria dos casos, os adolescentes constroem seu projeto apesar das dificuldades que encontram, implicando que tenham de lidar com as relações objetais, a fim de abandonar gradualmente a posição infantil. Para todos os jovens, observam-se momentos de avanços do projeto para o status de adulto (tempo de progressão), seguidos por outros momentos de retração, mudança ou pausa mais ou menos prolongada desse projeto (tempo de retração). Pode-se inferir a presença de um movimento de natureza oscilatória entre os dois polos das relações supracitadas, alternando os movimentos progressivos [progrédients] e regressivos [régrédients].

Esse movimento é explicável pela presença da angústia do futuro e de suas incertezas, mas também, no nível das relações, pelo que é vivenciado como demasiadamente próximo do "outro", por exemplo, com o outro sexo, numa proximidade que pode ser percebida e vivenciada como insuportável, porque o sujeito ainda não é capaz de assumir as consequências (i.e., não é capaz de imaginar a possibilidade de modificar as relações com o outro sexo adotando uma nova posição relacional, que seria então fundada em relações de complementaridade, não mais na existência de simples diferenças).

Por outro lado, esse movimento pode ser explicado pelo que é experimentado como grande distância com o "outro", especialmente porque essa distância ajuda a manter o jovem em um estado infantil, cuja inadequação é testemunhada por seu corpo, o que lhe é insustentável (CHABERT, 1993).

Isso também significa que o trabalho de construção do projeto não é estritamente linear, diferentemente do que pensa e aplica certo número de atores profissionais ou pais. Esses movimentos oscilatórios permitem uma integração gradual dos dados concernentes às relações do jovem, tanto com ele como com os outros e com o seu ambiente social e cultural.

\section{Cultura(s) e a Construção do Projeto Adolescente}

Em uma sociedade em constante movimento, em uma globalização baseada em movimentos migratórios, mas também por ser um importante desafio social, é essencial abordar a questão da construção do projeto de adolescentes cujos pais ou avós migraram. Para ilustrar isso, vamos abordar o caso de jovens cujos antepassados, marroquinos, estabeleceram-se na França.

\section{Um contexto}

A ideia de projeto é relativamente nova no Marrocos (RIARD; DACHMI, 2004; RIARD, 2005), pois, até recentemente, o filho recebia sua profissão de seu pai e, portanto, não tinha a oportunidade de expressar seus próprios desejos. É nesse contexto que os pais ou avós desses jovens chegaram à França. Para tais jovens, mesmo que o trabalho psíquico de remanejamentos seja substancialmente comparável ao 
observado em outros adolescentes, a construção de seu projeto na chamada sociedade anfitriã coloca-os diante de dificuldades adicionais, algumas delas inerentes ao teor de seu patrimônio cultural, introduzindo diferenças que podem ser vivenciadas como danosas em relação à juventude nativa.

\section{A herança cultural e seus componentes}

Todos os jovens têm de lidar com a sua história e com a história de seus pais (o que lhes foi dado, o que eles retiveram e o que rejeitaram; CICCONE, 1997). É essa herança que, de acordo com KAËS, "conecta o indivíduo ao social, e lhe permite codificar e decodificar as representações e os afetos organizados com maior ou menor flexibilidade em uma dada área cultural e para um sujeito singular" (KAËS, 1979, p. 26). A herança é composta por códigos relativos aos valores, às formas de significar objetos, aos modos de ser relacionar com os outros, às representações de homem e de mulher, assim como ao interesse atribuído à escola, ao sentido de honra, ao lugar dado ao tempo ou também à religião e à tradição.

Todas essas variáveis estão presentes, de alguma maneira, no projeto dos jovens. \#elas ajudam a desenhar seu contorno e qualidade, além do fato de que a observância dessas variáveis garante a segurança dos jovens na cultura que as contém. Essa herança é necessariamente mais pesada e difícil de assumir para os jovens cujos pais ou avós migraram, por causa da multiplicidade de referências culturais e, portanto, de escolhas a serem feitas (RIARD; ALAGUI, 2013).

\section{Repercussões da cultura sobre o projeto}

\section{Repercussões para os jovens}

Estabelecidos em uma área cultural diferente da de seus antepassados, muitos jovens sentem-se desconfortáveis com a totalidade ou com parte de sua herança, já que seu projeto obriga-os a fazer uma escolha de códigos ou partes de código; ou seja, a escolher entre os propostos pela cultura de seus antepassados e aqueles propostos pela cultura do país em que nasceram. Muitos jovens estão, assim, divididos entre sua fidelidade à cultura dos seus antepassados e o que deriva de sua própria experiência, adquirida na escola, em associações e pelos os meios de comunicação do país em que vivem.

Como resultado, alguns desses jovens estão sujeitos a tensões severas. que geram um mal-estar em geral originado em conflitos para os quais nem sempre encontram uma saída. Essas tensões também podem decorrer de uma inversão, frequentemente observada, das representações das suas relações com o outro sexo, em comparação com as dos jovens europeus; ou, ainda, de uma pobreza do ideal do eu, que é reconhecível pela pobreza de sua projeção no futuro, especialmente em termos de ideais pessoais. Por fim, tais tensões também podem decorrer da grande separação ou mesmo clivagem observada entre os universos da família e da escola (RIARD; PEYRE, 1997; RIARD, 2011). Quando há uma mudança de código (ver adiante), essas dificuldades não apenas geram mal-estar, mas também sentimento de insegurança e de culpa, pelo fato de tais mudanças serem consideradas transgressões em relação à cultura de suas origens, infidelidade a ela.

\section{Repercussões na família e seu entorno}

A escolha dos códigos, realizada pelos adolescentes, repercute também em suas relações com seus pais e levanta, principalmente, a questão da relação entre filiação e afiliação (KAËS, 1985). Assim, quando os códigos adotados pelos jovens são próximos daqueles de origens ancestrais, o jovem se inscreve em uma filiação, em uma continuidade de gerações, e o pai desse jovem fica, então, em consonância com sua 
comunidade que o reconhece como "bom pai” (MEDEJEL, 1998). Por outro lado, quando o jovem escolhe códigos culturais afastados daqueles de seus antepassados, filiando-se a outros valores, quando há mudanças significativas de código entre duas gerações, o jovem não se coloca apenas ele mesmo em "perigo" em suas relações com sua família, mas essa última também se vê em risco, porque o pai não pode mais pensar em seu filho com base em uma continuidade cultural ,o que trás a ameaça de não ser reconhecido como "bom pai" por sua comunidade, por não ter sido "capaz" de manter seu filho na cultura da família.

Assim, alguns desses jovens usam estratégias diferentes para construir seu projeto, sem as quais tal projeto não seria possível.

\section{O Lugar do Setor Associativo}

Alguns jovens que não se beneficiam - ou se beneficiam insuficientemente - dos aportes da escola ou não têm ajuda de suas famílias nesse processo e se sentem incapazes de fazer as mudanças necessárias para a construção de seu projeto direcionam-se para associações de bairro (associações comunitárias), como é o caso dos jovens de que tratamos anteriormente. 5

Os setores associativos (associações comunitárias) têm características diferentes das dos dispositivos educativos convencionais. Elas não impõem presença regular; os jovens as frequentam de modo voluntário, por períodos variáveis que vão de passagens efêmeras à frequência durante alguns anos. Além disso, as relações entre os membros e com os profissionais baseiam-se numa certa liberdade de trocas.

Partimos da hipótese de que essas associações (por exemplo, os centros sociais) constituem espaço de criatividade, cujas características se assimilam às da análise transacional, no sentido proposto por KAËS (1979). É possível considerar, com efeito, que elas constituem uma "zona intermediária de experiência" (entre várias culturas), e "que promovem um processo de passagem (transição) entre dois estados subjetivos, constituindo uma área na qual se elabora a questão do código cultural” (KAËS, 1979, p. 60).

Nessas estruturas, mais flexíveis do que as dos organismos sociais comuns, como a escola, os jovens são colocados sob o olhar dos animadores (na maioria das vezes jovens adultos também de outras culturas), que podem ser considerados ocupantes de uma função particular para o jovem, na medida em que essa relação chega em um momento oportuno para ele; ou seja, de acordo com seu caminho pessoal, modificando assim a sua relação com o seu grupo e com ele mesmo, no sentido de reforçar sua segurança interna. É possível considerar, então, que os animadores ocupem uma função de "outro educador" [tiers éducatif]. Além disso, a presença de outros adolescentes, oriundos também de outras culturas, multiplica não só as possibilidades de encontros, trocas, conexões, mas oferece, por isso mesmo, uma diversidade de códigos culturais, o que é propício à criatividade.

No entanto, graças, na maioria das vezes, a uma série de representações errôneas de moradores do bairro em relação ao centro social ("não serve para nada"; "não propõe nada de interessante"; "é perigoso para as meninas”), alguns desses jovens não podem frequentá-lo regularmente, nem mesmo adentrá-lo, embora desejem fazê-lo. A despeito dessa interdição, uma parte desses jovens, provavelmente com maior motivação que outros, contando com a conivência de alguns animadores, ou mesmo se beneficiando da ajuda de companheiros que já tenham acesso ao equipamento, acabam participando de modo informal e pontual. Aconselhados e apoiados por um animador do centro, com o qual estabeleceram vínculo, desenvolvem então uma estratégia de persuasão junto ao pai, que, uma vez convencido, dispõe-se a encontrar o animador do centro social. Suficientemente aberto para o exterior, esse pai, incapaz de responder às demandas de seu filho quanto ao seu projeto, é capaz de compreender a importância de tais demandas, delegando assim, de certa 
maneira, uma parte da autoridade de pai para o animador, ao "entregar-lhe" seu filho para que o ajude na realização de seu projeto (RIARD, 2011).

Nessas circunstâncias, a associação ocupa uma função de espaço intermediário, capaz de articular, reduzir ou facilitar a aceitação do impacto das diferenças entre pai e filho, permitindo conciliar os termos da contradição que atravessa ambos. Nossa hipótese é, então, que esse espaço social, ao oferecer ao jovem um escoramento, vem preencher uma lacuna em relação à impossibilidade de elaboração de um projeto, facilitando assim a transição de um código para outro, com base em uma aproximação entre as duas culturas. Tal espaço permite que o jovem vá mais longe no esforço de afiliação à cultura do país em que vive, ou a partes dela, e permite ao pai aceitá-la, reduzindo assim o risco de que a afiliação do jovem a certas partes da cultura do país de moradia conflite com sua própria filiação e a história de seus antepassados. Desse modo, a associação constitui uma zona intermediária (KAËS, 1979), um espaço que não pertence a uma cultura ou outra, mas que permite ao jovem, por meio dos apoios proporcionados por cada cultura, reorganizar-se e encontrar um novo escoramento. $\mathrm{O}$ animador, como representante da associação, ocupa então uma posição de intermediário, ao permitir que o pai possa lidar com os eventuais questionamentos de elementos de seu próprio patrimônio cultural, que implicam esaa "delegação".

Nessas condições, o centro social aparece como uma terceira via, ao lado da escola e da família, que permite a codificação de novas práticas, desempenhando o papel de tratamento, transformação e, em última análise, aceitação de diferenças, e permitindo, assim, a codificação de novas práticas, relacionadas ao projeto. Esse espaço vem para preencher um sentimento de falta em relação ao projeto, o qual se via impossibilitado na situação em que se encontrava o sujeito.

\section{Conclusão}

No final destas apresentação e análise, necessariamente reduzidas, dos três elementos que consideramos marcos da construção do projeto, ressalta-se a presença de uma complexidade muito maior do que o suposto pelas práticas comumente propostas aos adolescentes no âmbito das orientações escolar e profissional dispensadas pela escola, construção essa que não poden ser restringida unicamente a dimensões cognitivas e intelectuais. Essa abordagem restrita pode provocar uma clivagem dentro dos próprios jovens, podendo levar alguns a construírem seu projeto de acordo com o desejo dos outros ou, no mínimo, gerando profunda insatisfação quando os jovens vierem a descobrir, já engajados na vida adulta, que seu projeto não é, de maneira alguma, seu projeto, que sua atividade profissional não corresponde a escolhas reais e que, apesar das aparências, sua vida privada não foi necessariamente escolhida, mas determinada pelos outros. Se a dimensão afetiva não pode prevalecer, ela tampouco pode ser negligenciada ou obscurecida, o que confirmam muitas terapias adultas.

\section{REFERÊNCIAS}

BLOS, P. Les adolescents: Essai de psychanalyse. Paris: Stock, 1967.

CASTELLAN, Y. Adolescence, rupture et continuité. Carrefours de l'Education, v. 24, p. 21-35, 2007. https:// doi.org/10.3917/cdle.024.0021

CASTELLAN, Y.; RIARD, E. H. Les 12-17 ans: Le projet de vie et ses voies. Carrefours de l'Education, v. 19, 
p. 139-164, 2005. https://doi.org/10.3917/cdle.019.0139

CHABERT, C. Modalités du fonctionnement psychique des adolescents à travers le Rorschach et le TAT. Psychologie Française, v. 28, n. 2, p. 187-194, 1993.

CICCONE, A. Empiètement imagoïque et fantasme de transmission. In: EIGUER A. (org.). Le générationnel, approche en thérapie familiale psychanalytique. Paris: Dunod, 1997, p. 153-185. (Collection Inconscient et Culture).

CRITES, J. O. Parental identification in relation to vocational interest development. Journal of Educational Psychology, v. 53, n. 6, p. 262-270, 1962. https://doi.org/10.1037/h0040197

ERIKSON, E-H. Adolescence et crise: La quête de l'identité. Paris: Flammarion, 1968.

GINZBERG, E.; GINSBURG, S.; AXERALD, S.; HERMA, C. Occupationnal choice. New York: Columbia University Press, 1951.

GOLSE, B. Le développement affectif et intellectuel de l'enfant. 4. ed. Issy-les-Moulineaux: Masson, 2008.

GUICHARD, J. Lécole et les représentations d'avenir des étudiants. Paris: Presses Universitaires de France, 1993.

GUTTON, P. Du changement à la puberté. Adolescence, v. 1, n. 1, p. 7-11, 1983.

HOANG, G. T. Construction du projet de vie chez les adolescents âgés de 15 à 17 ans scolarisés à Hanoi, Vietnam. 2012. Thèse. (Doctorat en Psychologie Sociale Clinique) - Faculté de Philosophie, Université de Picardie Jules Verne, Amiens, 2012.

HUTEAU, M. La psychologie du projet. ADAPT, n. 3, p. 7-13, 1993.

JEAMMET, P. Du familier à l'étranger. Territoires et trajets de l'adolescent. Neuropsychiatrie de l'Enfance, v. 31, n. 8-9, p. 361-381, 1983.

KAËS, R. Introduction à l’analyse transitionnelle. In KAËS (org.). Crise, rupture et dépassement. Paris: Dunod, 1979, p. 1-81.

KAËS, R. Filiation et affiliation. Gruppo, v. 1, n. 1, p. 23-45, 1985.

KAËS, R. L’idéologie, l’idéal, l’idée, l’idole. Paris: Dunod, 2016.

KESTEMBERG, E. L'identité et l'identification chez les adolescents. Problèmes théoriques et techniques. Psychiatrie de l'Enfant, v. 5, n. 2, p. 441-522, 1962. https://doi.org/10.3917/dunod.chagn.2012.02.0323

LESOURD, S. La construction adolescente. Toulouse: Eres, 2005. 
MAHLER, M. Psychose infantile. Paris: Petite Bibliothèque Payot, 1982.

MAINE, J. Estime de soi et parrainage chez les jeunes de 14 à 17ans: De la logique d'assistance à la logique d'investissement. Le rôle d'un livret d'épargne. 2009. Thèse. (Doctorat en Psychologie Sociale Clinique) Faculté de Philosophie, Université de Picardie Jules Verne, Amiens, 2009.

MÂLE, P. La crise juvénile. Paris: Payot, 1962.

MARCELLI, D.; BRACONNIER, A. Adolescence et psychopathologie. 6. ed.. Issy-les-Moulineaux: Masson, 2007.

MEDEJEL, M. Quelques questions posées à propos de l'éloge des grands frères. In: RASSIAL, J. J. (org.). Y-a-t-il une psychopathologie des banlieues? Toulouse: Eres, 1998. https://doi.org/10.3917/eres.rassi.2002.01.0107

MURRAY, H. A. Exploration de la personnalité. Paris: Presses Universitaires de France, 1953.

RIARD, E. H. Risque d'errance à l'adolescence: Proposition de l'hypothèse de l'oscillation polarisée, Pratiques Psychologiques, v. 1, p.13-21,1996.

RIARD, E. H. Les jeunes et leur projet de vie dans les contextes français et marocains, Journal des Psychologues, n. 233, p. 45-49, 2005.

RIARD, E. H. Place des associations dans l'émergence du projet de vie des jeunes issus du Maroc résidant en Picardie (approche qualitative). In: MELYANI, M. /Ingénierie du lien social: Développement et associations. Amiens: Licorne, 2006.

RIARD, E. H. Entre deux cultures. Les associations de quartiers comme tiers éducatif pour des jeunes issus de l'émigration. In: XYPAS, C.; FABRE, M.; HETIER, R. Le tiers éducatif, une nouvelle relation pédagogique. Figures et fonctions du tiers en éducation et formation. Bruxelles: De Boeck, 2011, p. 137-152. https://doi. org/10.3917/dbu.xypas.2011.01.0137

RIARD, E-H., PEYRE, V. Etude des facteurs de risque de marginalisation d'une population de 12 à 16 ans de la Région drouaise dans la dialectique individu-société. (Protection Judiciaire de la Jeunesse, Eure et Loir), (Rapport de recherche). 1997.

RIARD, E-H.; DACHMI, A. Adolescence et projet de vie (Approche de psychologie clinique et sociale), Publication de la Faculté des Lettres et des Sciences Humaines, Rabat, Essais et études, n. 40, 2004.

RIARD, E-H.; ALAGUI, A. Projet professionnel d'étudiants marocains en France issus de la migration: Rôle de la famille et de l'université. In: VATZ LAROUSSI, M.; RIARD, E-H.; GELINAS, C.; JOVELIN, E. (org.). Les défis de la diversité. Paris: L’Harmattan, Espaces interculturels, 2013, p. 301-313

SUPPER, D. E. Travail et loisirs dans une économie en flux. L'orientation Scolaire et Professionnelle, v. 17, n. 1, p. 23-32, 1988. 


\section{Notas}

1. Baseadas em contratos associando o departamento de Eure e Loir, o Ministério da Justiça e a Proteção Judiciária da Juventude (RIARD; PEYRE, 1996); uma Mission Locale em Île-de-France (CASTELLAN; RIARD, 2005; CASTELLAN, 2007); ou ainda no âmbito de ações integradas (Programa Hubert Curien) do Ministério das Relações Exteriores (RIARD; DACHMI, 2004; RIARD; ALAGUI, 2013).

2. Esses trabalhos constituiram ponto de partida importante para a elaboração de um procedimento de identificação das primeiras dificuldades dos jovens entre 12 e 14 anos (não considerado aqui - Projeto Regional Estruturante Picardia), estabelecido com base numa amostra representativa de 2.300 sujeitos, no que tange às 57 questões relativas às dimensões psicossocial e psicoafetiva da prova. (Esse procedimento inclui também as dimensões psicomotora, cognitiva e intelectual.)

3. A instrução dada no início, conforme são distribuídas as cartas selecionadas, é: “imaginem uma história dizendo o que se passou antes, o que se passa agora e como a história vai terminar. Para cada personagem da historia, diga também o que ele pensa e o que ele sente". Quanto às cartas selecionadas, foram apresentadas na seguinte ordem: carta 2 (pais) para todos os sujeitos; carta 6 (relações mãe/filho - num contexto de mal-estar; carta mãe/filho - concebida e validada segundo o modelo da carta destinada aos meninos); carta 7BM (relações pai/meninos; relação pai/meninas) concebida (e validada) segundo o modelo da carta destinada aos meninos; carta 4 (relações com os pares) para meninos e meninas. Por fim, apresentação da carta 16 (destinada a ambos os sexos).

4. O que entendemos aqui por "setor associativo" recobre o conjunto de estruturas dirigidas aos jovens (maisons de quartier, maisons des jeunes et de la culture, centres sociaux, mission locale etc.), cuja frequência é livre. As atividades propostas podem ser de um único tipo (por exemplo, acompanhamento escolar) ou variadas, indo do acompanhamento escolar às atividades culturais e esportivas. As atividades são conduzidas por adultos profissionais (animadores) ou voluntários. As fontes de financiamento dependem de diferentes instâncias do poder público (Estado, município, governo regional etc.).

5. Não incluímos em nossa exposição os jovens que frequentam as associações exclusivamente destinadas ao "acompanhamento escolar", pois em geral o fazem por determinação dos pais (RIARD, 2006)

Recebido: 01 Mar 2019

Aceito: 17 Set 2019

Comitê editorial Cedes/Coordenação deste número:

Izabel Galvão e Maria Rosa Camargo 